\title{
Epidural Corticosteroids, Lumbar Spinal Drainage, and Selective Hemodynamic Control for the Prevention of Spinal Cord Ischemia in Thoracoabdominal Endovascular Aortic Repair: A New Clinical Protocol
}

\author{
Alberto Pasqualucci · Ayman Al-Sibaie · Kurian Palavilayil Thomas Vaidyan · Antonella Paladini · \\ Mansour Yousef Nadhari · Fabio Gori · Hany Fawzy Greiss · Marina Properzi · Osama Sami Maki Al Ani • \\ Aruna Godwin · Altaf Hussein Syedkazmi · Osama Ahmed Elhanf · Giustino Varrassi if \\ Received: August 30, 2019 / Published online: November 12, 2019 \\ (C) The Author(s) 2019
}

\section{ABSTRACT}

Introduction: In patients undergoing thoracoabdominal aorta repair, spinal cord ischemia (SCI) remains one of the most common and important complications resulting in transient paraparesis through to permanent flaccid paraplegia. In this manuscript, after a brief introduction to spinal cord ischemia complication

Enhanced Digital Features To view enhanced digital features for this article go to https://doi.org/10.6084/ m9.figshare.10110695.

Electronic supplementary material The online version of this article (https://doi.org/10.1007/s12325019-01146-7) contains supplementary material, which is available to authorized users.

A. Pasqualucci · F. Gori · M. Properzi

Department of Surgical and Biomedical Science,

University Hospital of Perugia, 06100 Perugia, Italy

A. Pasqualucci - A. Al-Sibaie - K. P. T. Vaidyan · M. Y. Nadhari - H. F. Greiss - O. S. M. Al Ani . A. Godwin · A. H. Syedkazmi · O. A. Elhanf Rashid Hospital, Trauma and Emergency Center (Dubai Health Authority), Dubai, UAE

A. Paladini

Department of MESVA, University of L'Aquila, 67100 L'Aquila, Italy

G. Varrassi $(\bowtie)$

Paolo Procacci Foundation, Via Tacito 7, 00193 Rome, Italy

e-mail: giuvarr@gmail.com and its prevention in thoracoabdominal endovascular aortic repair (TEVAR), we propose a new clinical protocol potentially able to prevent such complication.

Methods: The proposed protocol suggests the use of high dosages of corticosteroids by epidural route, along with drainage of cerebrospinal fluid and controlled vascular hypertension, to reduce the incidence of SCI in TEVAR. Moreover, we paid particular attention to the control of the hemodynamic parameters to obtain adequate peripheral tissue perfusion (oxygen delivery), including in the spinal cord. Results: We applied this new protocol in 50 consecutive patients treated with TEVAR for thoracoabdominal aortic aneurysms (TAAs); 47 patients completed the procedure: 27 patients Crawford type I and 20 Crawford type II. Three patients died during surgery because of untreatable aneurysm rupture. The results show that in all patients there were no cases of SCI, after 5 days from TEVAR.

Discussion: To the best of our knowledge, there are no clinical studies on the use of epidural corticosteroids in patients undergoing treatment of aortic syndrome (both in "open surgery" and endovascular aortic repair). This initial study on 50 consecutive patients has shown that the clinical protocol used could be of great interest to prevent one of the worse complications of TEVAR. Its limitations are the low number of patients studied till now, and the 
non-randomized protocol adopted. Further studies would be necessary.

Conclusion: Our experience and the results obtained with this new perioperative protocol with epidural corticosteroid and accurate hemodynamic control have been encouraging and it seems a valid proposal to be explored in future by well-structured prospective, randomized protocols.

Keywords: Cerebrospinal fluid drainage; Corticosteroids; Epidural corticosteroids; Goaldirected hemodynamic therapy; Hemodynamic; Methylprednisolone; Spinal cord ischemia; Thoracoabdominal endovascular aortic repair

\section{Key Summary Points}

This study presents a new protocol as prophylaxis for the ischemic damage of the spinal cord during thoracic/ abdominal aortic repair.

The literature is rich in proposals, but none is really innovative and definitive. This research proposes the introduction of a new concept: the use of high dosages of epidural steroids. This, together with other preventive therapy, has prevented any neurological damage to the spinal cord in the 50 cases studied.

The results obtained with this new protocol are stimulating in a challenging topic that does not have any definitive answer, till now. It should be of interest for other research groups studying patients with aortic syndrome necessitating thoracoabdominal aortic repair.

\section{INTRODUCTION}

Unlike aortic surgical repair confined to the abdominal cavity, thoracoabdominal aortic aneurysm (TAA) repair poses substantial risks to patients and challenges to clinicians. Without surgery, the prognosis for patients with TAA damage remains poor. Since the incidence of TAA disease increases with aging, clinicians in the future will likely be facing increasing numbers of such procedures in many countries with growing elderly populations. Increasing the challenge, these surgical procedures can be more difficult to perform in geriatric patients. Both open TAA repair and newer endovascular approaches (including so-called hybrid procedures) are associated with a high rate of serious complications, including mortality, stroke, renal failure, respiratory insufficiency, and spinal cord ischemia (SCI) [1-5]. In the past, the incidence of SCI was as high as 30\% when the entire thoracoabdominal aorta was resected [6]. Moreover, the rate of complications is not always lower in hybrid endovascular repairs compared to open procedures $[7,8]$. Indeed, the incidence of SCI has not diminished with the advent and improvement of hybrid and endovascular techniques $[9,10]$. Thus, SCI remains a serious and unresolved problem $[1-3,11-20]$.

Because of the vascularization of the spinal cord (Fig. 1), SCI may result in transient paraparesis of varying degrees or permanent flaccid paraplegia [1, 3, 11, 21-23]. Immediate, proactive, and aggressive strategies may be able to successfully treat SCI as there may be an early window of time in which certain forms of SCI may be reversible [9], but it should be better prevented. A variety of protective strategies have been reported, such as hemodynamic control, drainage of cerebrospinal fluid (CSF), and neuroprotective pharmacotherapeutic agents $[6,22,24]$.

Various protocols have been employed to prevent SCI during thoracoabdominal endovascular aortic repair (TEVAR), in which the most important concepts are CSF drainage, controlled arterial pressure, and the use of neuroprotective drugs, e.g., corticosteroids. Drainage of CSF and controlled arterial pressure are typically used together to improve spinal cord perfusion and prevent SCI during interventions. Perioperative lumbar drainage of CSF has been widely used in open aortic surgery but has also been applied to TEVAR. The lumbar 


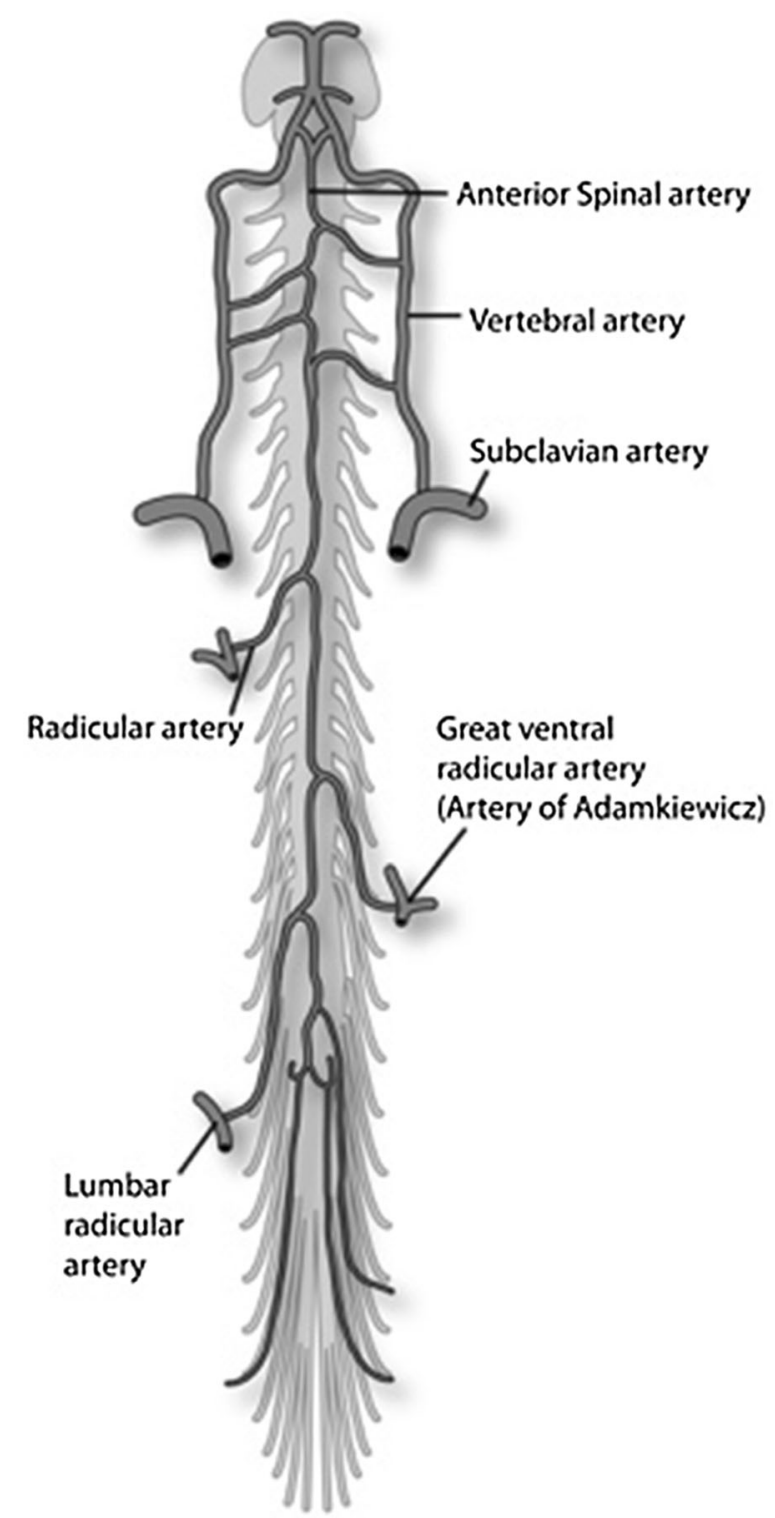

Fig. 1 Arteries around the spinal column (Art work courtesy of Todd Cooper)

catheter's position in the subarachnoid space for monitoring CSF pressure and draining CSF plays a key role in SCI prevention. CSF drainage and monitoring are based on a physiologic principle. Spinal cord perfusion pressure (SCPP) may be defined as the difference between the mean arterial blood pressure (MAP) and the CSF pressure (CSFP) [13]: SCPP $=$ MAP - CFSP. During aortic surgery, CSFP is reduced (approx. $10 \mathrm{mmHg}$ or $13 \mathrm{cmH}_{2} \mathrm{O}$ ) by draining the CSF and, at the same time, controlling arterial pressure (maintaining a high MAP $\geq 85 \mathrm{mmHg}$ ) such that SCPP is greater than $75 \mathrm{mmHg}$. CSF drainage may also remove negative neurotrophic factors, such as tissue-type plasminogen activator (tPA) and others, which are false transmitters that can result in ischemic damage $[13,15]$.

While some literature has confirmed that these techniques result in a reduced incidence of SCI in both open surgery $[13,16,17,25]$ and TEVAR $[1,11,15,16,25-27]$, other studies are inconclusive $[1-3,11]$. A systematic review reported that prophylactic CSF drainage resulted in an SCI rate during TEVAR of 3.6-6.8\% [25]. A recent meta-analysis reported that 9-13\% of patients had SCI following TEVAR $[15,28]$, but other authors reported that the incidence of post-TEVAR SCI was approximately $3.9 \%[1,25,26,28]$. A significant reduction in major complications in the treatment of Crawford type I-II and III [29] TAAs was observed when patients were subjected to aggressive preoperative and postoperative protocols (blood and platelet transfusions, $\mathrm{MAP}>85 \mathrm{mmHg}$ with CSFP $\leq 10 \mathrm{mmHg}$, neuroprotective agents, etc.) aimed at optimizing spinal cord perfusion. The risk of SCI decreased from approximately $13-14 \%$ down to $1.2-3.7 \%[1,11,16,25-28]$. Immediate reversal of neurological deficit has likewise been reported in open surgery when CSF fluid is drained via a spinal catheter following thoracic aortic repair $[1-3,11,16]$. In fact, CSF drainage has been shown to reduce the incidence of delayed paraplegia in endovascular procedures $[1,3,11,16,29]$. This bolsters the argument that CSF drainage is an important factor in reducing the incidence of SCI. CSF drainage should be based on CSFP monitoring, which should continue for about $48 \mathrm{~h}$ following the procedure. Incidence of neurological deficits during that time period may necessitate prolonged monitoring and drainage $[1,11,30]$. Hypotensive episodes during this period should be avoided [1, 3, 11-17, 25, 26, 28, 30]. Thus, it seemed important to evaluate whether these factors, namely the maintenance of CSFP (approx. $10 \mathrm{mmHg}$ ), CSF drainage, and MAP $>80$ or $90 \mathrm{mmHg}$, would be able to maintain spinal cord perfusion pressure at $75 \mathrm{mmHg}$ or higher in such a way that it reduced the risk of SCI. 
When blood flow to the spinal cord is interrupted, many localized events occur, such as the production of free radicals, the accumulation of toxins [31], the increased concentration of the excitatory amino acid glutamate [32], and so forth, any of which can cause secondary cell damage and death. Free radicals can cause ongoing cell damage even after blood supply to the spinal cord is restored [31]. Corticosteroids, in particular methylprednisolone, have been investigated for their mechanism of action and effectiveness in preventing or treating SCI. In addition to anti-inflammatory action, methylprednisolone improves spinal cord blood flow and microvascular perfusion [33-35], an effect known and confirmed for about half century [36]. It provides cytoprotection by inhibiting oxygen free radical-induced lipid peroxidation, which occurs in degenerative events [37, 38]. Methylprednisolone may also protect against secondary neuronal degeneration and reduce the accumulation of intracellular calcium [37]. Data from the National Acute Spinal Cord Injury Study (NASCIS) II and III have demonstrated a moderate benefit from parenteral administration of high-dose methylprednisolone when administered within $8 \mathrm{~h}$ of injury $[39,40]$. There is ongoing debate over the validity and clinical applicability of the NASCIS studies, since such high doses of these drugs are associated with potentially serious adverse events [41-45]. Koszdin et al. [46] reported in animal studies that there was poor spinal bioavailability of methylprednisolone following systemic administration, presumed to be related to P-glycoprotein-mediated exclusion of methylprednisolone from the spinal cord. Since the process of secondary damage begins within minutes of the primary insult of SCI, there is little time for detection and effective management once secondary injury mechanisms set in. For that reason, interventions must be carried out as early as possible [47-49]. The aforementioned complications may be reduced by prophylactic and localized administration of corticosteroids (by epidural or intrathecal route), thereby resulting in maximal immediate steroid availability and effects, and eliminating potential systemic complications [28]. Intrathecal administration of drugs can reduce the dosage substantially and, in that way, reduce side effects, while still exerting a direct steroidal effect on spinal cord tissue (significantly decreasing ischemia-induced local events by direct inhibition) [32, 50]. Epidurally administered drugs can traverse meningeal layers and achieve significant concentration in the CSF [51]. Quirno et al. [50] have shown in animal studies that prophylactic epidural administration of high-dose corticosteroid therapy can provide effective neuroprotection against secondary tissue damage. Epidural corticosteroids may be particularly beneficial, although there is a paucity of literature on the potential role of these agents to help prevent SCI. Although there are no clinical studies to date on the use of epidural corticosteroids in patients undergoing procedures for aortic syndrome, the authors have decided to adopt a new clinical protocol to prevent SCI during TEVAR, which would provide CSF drainage, control the MAP, and use epidurally administered corticosteroids. The rationale behind using epidural administration of methylprednisolone is based on the literature: epidural administration of methylprednisolone, in contrast to systemic delivery, is effective in reducing spinal cord inflammation [16, 32, 46, 49-51]. Moreover, this new clinical protocol would provide greater attention to the control of hemodynamic parameters, always important in surgeries even partially involving the aorta [52] in particular, offering a "how to" in achieving and maintaining the desired MAP ( $\geq 85 \mathrm{mmHg}$ ) by optimizing peripheral tissue perfusion (oxygen delivery) based on goal-directed hemodynamic therapy (GDT) [53-60].

\section{METHODS}

This protocol was used in the first 50 consecutive patients treated with TEVAR for TAAs with Crawford classifications type I and II (both acute and non-acute) from July 2016 to November 2018 at two important teaching hospitals ("Santa Maria della Misericordia" University Hospital, Perugia, Italy; and Rashid Hospital, DHA, Dubai, UAE). The clinical protocol was internally approved by the Dubai Health Authority (see supplementary material). 
Considering that it was just proposing a different way of administration of well-established drugs, approval of the ethics committee was not necessary. All the patients signed an informed consent, specifically indicating that they would have been administered epidural instead of systemic steroids, beside drainage and pressure monitoring of the spinal fluid. They also accepted that their cases could be published, preserving their privacy. The study has been conducted in accordance with the Helsinki Declaration 1964 and its later amendments.

\section{Clinical Protocol}

Before any anesthesiologic approach, basic monitoring was carried out in all the patients (ECG, invasive blood pressure, central venous pressure, temperature, etc.). The optimal site for the epidural injection of methylprednisolone and CSF drainage is between the first and fourth lumbar intervertebral spaces $\left(\mathrm{L}_{1}-\mathrm{L}_{2}-\mathrm{L}_{3}-\mathrm{L}_{4}\right)$. Using a subarachnoid spinal catheter kit for pressure measurement, the anesthetists inserted a Tuohy needle (14 or 16 gauge) into a conscious, cooperative patient for the injection of corticosteroid (see Fig. 2 and Table 1), either in the interspace $\mathrm{L}_{1}-\mathrm{L}_{2}$ or $\mathrm{L}_{2}-\mathrm{L}_{3}$, with the patient lying in a lateral position. The epidural administration of methylprednisolone occurred at a level slightly higher than the CSF drainage level. Using a "loss of resistance" technique, the anesthetist identified the injection site and $160-200 \mathrm{mg}$ of methylprednisolone acetate diluted in $25-30 \mathrm{ml}$ of saline solution was injected into the epidural space. The volume and dosage were determined on the basis of medullary segments involved (Crawford's classification) $[1,3,5,61-63]$, in order to cover all of the segments potentially involved in the vascular injury. After 5-10 min, the subarachnoid catheter for CSF drainage was placed via a 16-gauge spinal needle at $\mathrm{L}_{3}-\mathrm{L}_{4}$ or $\mathrm{L}_{4}-\mathrm{L}_{5}$. Once the position in the subarachnoid space was confirmed, a $50-\mathrm{cm}$ catheter was inserted to drain the CSF and continuously monitor CSF pressure. During the course of the procedure, the CSF pressure was continuously monitored and the physician had the possibility to decide

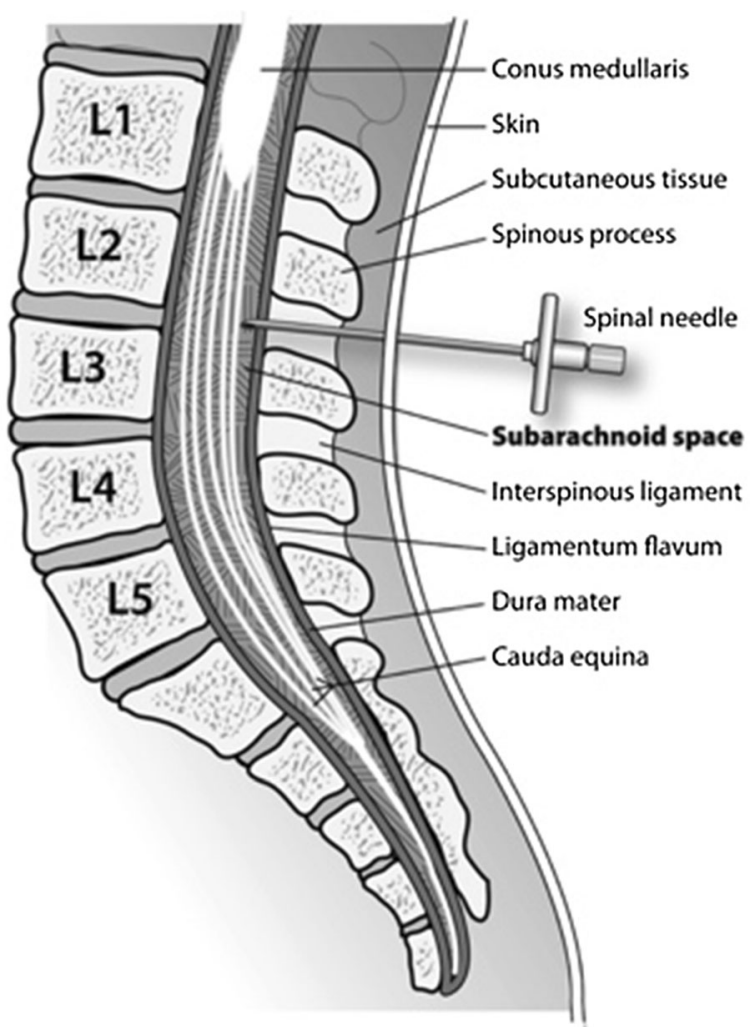

Fig. 2 Lumbar puncture (Drawing courtesy of Todd Cooper)

if and when to drain CSF in order to maintain a CSF pressure of approximately $10 \mathrm{mmHg}$ [2, 3, 11, 13, 17]. Before TEVAR was initiated, the patient underwent general anesthesia with orotracheal intubation. General anesthesia was maintained with continuous sevoflurane and remifentanil or with whatever drugs and method the anesthetist determined was most appropriate. In the immediate pre-procedure period, an echocardiogram was performed to optimize the patient's hemodynamic status (particularly preload, cardiac contractility status, volume of liquids to be infused, and so on).

The protocol is summarized in Table 1. Throughout the entire perioperative period, the following pressure values were maintained: spinal cord perfusion pressure at least 75-$80 \mathrm{mmHg}$; $\mathrm{MAP} \geq 85 \mathrm{mmHg}$; and CSF pressure $\leq 10 \mathrm{mmHg}$. Goal-directed hemodynamic therapy (GDT) was applied [18-20, 53-60]. GDT was based on optimizing preload with the use of algorithms that control fluids, inotropes, and/or 
Table 1 Summary of the protocol for perioperative management of patients undergoing TEVAR

Subject

Preoperative and intraoperative considerations

(a) Coagulation status

(b) Infection at site

(c) Intracranial process

Potential placement issues

Spinal pathology (stenosis)/prior lumbar spine surgery

\section{Recommendations}

Platelets $>100,000$

INR $<1.4$ and normal aPTT

Management of perioperative anticoagulants as per the institutional guidelines

Consider alternative site or delay surgery

Avoid drain placement if concern for intracranial hypertension

Consider imaging MRI or CT scan; allow for positioning limitations

Lumbar drainage insertion consider insertion prior to induction of anesthesia

(a) Injection of corticosteroid in epidural space followed by insertion of drainage catheter in the subarachnoid space

(b) Accidental initial lumbar puncture

Intraoperative management

(a) Hemodynamics
Best space between L1 and L4. When the needle is into epidural space, slowly inject a solution of 160-200 mg of methylprednisolone acetate diluted to $25-30 \mathrm{ml}$ in saline solution, depending on number of spinal segments involved

5-10 min after the injection in epidural space, a second needle is inserted into the subarachnoid space, at a lower intervertebral space, and a catheter is inserted $20-30 \mathrm{~cm}$ into the subarachnoid space to drain CSF and provide a continuous monitor of the CSF pressure

Evaluate for 24-h delay of surgery or anticoagulation management, as per the institutional guideline

Optimization of preload with $\mathrm{SV}>35 \mathrm{~mL} / \mathrm{min} / \mathrm{m}^{2}$; $\mathrm{CI}>2.5 \mathrm{~L} / \mathrm{min} / \mathrm{m}^{2} ; \mathrm{DO}_{2} \mathrm{I} \geq 600 \mathrm{~mL} / \mathrm{min} / \mathrm{m}^{2}$ $\mathrm{SvcO}_{2}>70-75 \%$ after endograft. Avoid hypotension Optimize MAP $>85 \mathrm{mmHg}$ with fluids and vasoactive support (dobutamine and noradrenaline). Blood and platelet transfusions: $\mathrm{Hb}>10 \mathrm{~g} / \mathrm{dl}$, platelet $>10^{5} / \mathrm{mL}$, plasma transfusion $10-15 \mathrm{ml} / \mathrm{kg}$ 
Table 1 continued

\section{Subject}

(b) Intraoperative, CSF drainage (monitor CSF drainage and calculate SCPP)

Postoperative management and monitoring

(a) Disposition

(b) Early neurological exam

(c) Position

(d) Neurological checks

(e) CSF drainage (monitor CSF drainage and calculate SCPP)

(f) Duration of CSF monitoring/drainage

(g) Hemodynamics

\section{Recommendations}

Optimize/maintain SCPP > $75 \mathrm{mmHg}$ : CSFP $\approx$ $10 \mathrm{mmHg}$ and $\mathrm{MAP}>85 \mathrm{mmHg}$

Avoid large volumes of CSF drainage (drain $<40-50 \mathrm{ml} / \mathrm{h}$ ), and drainage $=150-200 \mathrm{ml} /$ day

If there are no neurologic symptoms, it is not necessary to increase the drainage

Intensive care unit

Allow for fast-track anesthesia and early extubating for early evaluation

Elevate the head of the bed $20-30^{\circ}$ if there are no neurological deficits. If such deficits are present, let patient rest supine

Every $1 \mathrm{~h}$ for the initial $4 \mathrm{~h}$, then every $2 \mathrm{~h}$ for $8 \mathrm{~h}$, then every $4 \mathrm{~h}$ for $48 \mathrm{~h}$ if no neurologic change is noted

If there are no neurological symptoms, it is not necessary to increase the drainage

Optimize/maintain SCPP $>75 \mathrm{mmHg}$ : CSFP $\approx$ $10 \mathrm{mmHg}$ and $\mathrm{MAP} \geq 85 \mathrm{mmHg}$ for the first $12-24 \mathrm{~h}$ after the intervention

Maintain the CSF pressure $\leq 12 \mathrm{mmHg}$ for $12-24 \mathrm{~h}$ and $\leq 15 \mathrm{mmHg}$ for another $12-24 \mathrm{~h}$

Maximal drainage $=150-200 \mathrm{ml} /$ day

$<72 \mathrm{~h}$ to minimize infection risk

$48-72 \mathrm{~h}$ if neurologically intact and patient is Crawford type I or II

If central neurologic deficit is present, weigh the risk of infection versus benefit of continued CSF drainage

Avoid hypotension, augment BP if needed with vasoactive agents and fluids (see intraoperative) 
Table 1 continued

\section{Subject}

(h) Onset lower extremity neurological deficit

Neurologic Deficit Scoring system:

1, minimal or no motion: paraplegia

2, motion but not against resistance or gravity: paraplegia

3, motion against resistance and gravity but no ability to stand or walk: paraparesis

4, ability to stand and walk with assistance: paraparesis

\section{Recommendations}

Consider SCI vs neuraxial hematoma; call neurology and perform MRI or CT scan

Increase SCPP $>85-100 \mathrm{mmHg}$ : CSF pressure $<10 \mathrm{mmHg}$ (limit $=5 \mathrm{mmHg}$ ), increase MAP to $100 \mathrm{mmHg}$, up to $110 \mathrm{mmHg}$ (with intravascular volume expanders and pharmacologically) $[2,3,7,8,54]$

If there are neurologic symptoms, some authors reported drainages of more than $500 \mathrm{~mL} /$ day to achieve a CSFP $<10 \mathrm{mmHg}[2,3,8,12,16]$

Discontinuation of medications that could potentially interfere with neurological examinations

Clinical neurological examination for 5 days. Discontinue lumbar drain when neurological exam result plateaus or 5 days after procedure (whichever comes first) [26]

(i) Bloody CSF drainage

(j) Headache

(k) DVT prophylaxis

(l) Drain removal: Stable neurological exam after $6 \mathrm{~h}$ with drain clamp

Ensure intact catheter tip (rule out catheter fracture)
May indicate intracranial bleed, consider MRI or CT scan head and/or spine

Consider symptomatic intracranial hypotension and stop CSF drainage

Call neurology if neurological deficit appears (before resuming CSF drainage)

SQ heparin \pm SCDs

Ensure adequate coagulation profile similar to insertion guidelines

Delay removal $2-4 \mathrm{~h}$ after last heparin administration Hold heparin for $1 \mathrm{~h}$ after drain removal

$L M W H$ low molecular weight heparin, $S C P P$ spinal cord perfusion pressure, TAA thoracoabdominal aortic aneurysm, CSF cerebrospinal fluid, CSFP CSF pressure, MAP mean arterial blood pressure, SQ subcutaneously administered, SCDs sequential compression devices, $S V$ stroke volume, $C I$ cardiac index, $D O_{2}$ oxygen delivery

vasodepressors in order to achieve a specific goal in stroke volume (SV), cardiac index or cardiac output (CI or $\mathrm{CO}$ ), oxygen delivery $\left(\mathrm{DO}_{2}\right)$, central or mixed venous oxygen saturation $\left(\mathrm{SvO}_{2}-\mathrm{ScvO}_{2}\right)$ [53-60]. These parameters allow for improved fluid therapy and the administration of red blood cells or inotropes to ensure adequate perfusion and oxygenation of cells. To obtain these parameters with GDT, minimally invasive monitoring was used, with devices such as LiDCO $^{\mathrm{TM}}$ (LiDCO Hemodynamic Monitoring, Lake Villa, Illinois, USA) or Vigileo $^{\mathrm{TM}}$ (Edwards Lifesciences, Irvine, California, USA) systems that use a specific central venous catheter placement for measurement of $\mathrm{ScvO}_{2}[55,57-59]$. According to these protocols, to achieve a MAP $>85 \mathrm{mmHg}$, the following GDT indicators were followed:

- $\mathrm{SVI}>35 \mathrm{ml} / \mathrm{min} / \mathrm{m}^{2}$

- $\mathrm{CI}>2.5 \mathrm{l} / \mathrm{min} / \mathrm{m}^{2}$ 
- $\mathrm{DO}_{2} \mathrm{I} \geq 600 \mathrm{ml} / \mathrm{min} / \mathrm{m}^{2}$ or the best $\mathrm{DO}_{2} \mathrm{I}$ attainable

- $\mathrm{ScvO}_{2} / \mathrm{SvO}_{2} \geq 70 \%$ to $75 \%$

- $\mathrm{Hb}$ (hemoglobin) $\geq 10 \mathrm{~g} / \mathrm{l}$

- Ht (hematocrit) $>30 \%$

- Diuresis $>1 \mathrm{ml} / \mathrm{kg} / \mathrm{h}$

- Lactate $<2$ to $2.5 \mathrm{mmol} / \mathrm{l}$

These parameters helped to avoid perioperative coagulopathy and blood loss [13]:

- Prothrombin time $>50 \%$

- Plasma fibrinogen $>2 \mathrm{~g} / \mathrm{l}$ (fresh-frozen plasma was transfused at $10-15 \mathrm{ml} / \mathrm{kg}$ )

- Platelets > $100 \mathrm{~g} / \mathrm{l}$

After the TEVAR procedure (Table 1), the patients were awakened and extubated as soon as possible to assess neurological conditions. The patients were transferred to the intensive care unit (ICU) for continuous monitoring of physiological parameters and neurological conditions. Patients were carefully monitored and assessed every hour for the first $4 \mathrm{~h}$, then ever $2 \mathrm{~h}$ for the next $8 \mathrm{~h}$, and if no neurological changes were observed, every $4 \mathrm{~h}$ for $48 \mathrm{~h}$. If a change occurred compared to the patient's preoperative neurological status (noted in the first postoperative examination in the first hour after surgery), the patients were considered to have immediate SCI. Patients who experienced an interval of normal function postoperatively followed by a subsequent neurological deficit were deemed to have delayed SCI $[3,17,26]$. SCI is defined as any new lower-extremity motor or sensory deficit not attributable to other causes (such as epidural hematoma, intracranial pathology, peripheral neuropathy); SCI is further characterized as paraplegia or paraparesis on the basis of neurological deficit scoring (Table 2) [1, 3, 17, 23, 26].

If no neurological deficits developed in the first $48 \mathrm{~h}$, CSF drains were removed and hemodynamic monitoring discontinued. If neurological examination in the form of brain state and lower-limb assessment determined changes, the protocol required:

- Accelerated neurological assessment schedule (every hour)
Table 2 SCI scoring system

\begin{tabular}{ll}
\hline Score & Definition \\
\hline 1 & Minimal or no motion, paraplegia \\
2 & $\begin{array}{l}\text { Motion but not against resistance or gravity, } \\
\text { paraplegia }\end{array}$ \\
3 & Motion against resistance and gravity but no \\
& ability to stand or walk, paraparesis \\
4 & Ability to stand and walk with assistance, \\
& paraparesis \\
\hline
\end{tabular}

Note that scores 1-3 are used in the initial postoperative period because ambulation cannot be tested (score 4) until clinically appropriate

- Increase of SCPP > 85 to $100 \mathrm{mmHg}$ in order to maintain CSF pressure $<10 \mathrm{~mm} \mathrm{Hg}$ (with a limit of $5 \mathrm{mmHg}$ )

- Magnetic resonance imaging (MRI)

The lumbar drain was removed when the results from the neurological examinations plateau or at 5 days, whichever comes first. This is accepted as the time limit for possible neurological consequences due to TEVAR [17]. All the patients of this study have been controlled 30 and 90 days after surgery.

\section{RESULTS}

Three patients with complicated acute TAA, who had accepted to be treated with the proposed protocol, died at the beginning of surgery because of untreatable aneurysm ruptures. A total of 47 patients completed the procedure; their age range was 54-83 (mean age 70.1 years), 35 were men and 12 women. Twenty-seven patients were Crawford classification type I ( 21 men, 6 women) and 20 were type II (14 men, 6 women). MAP equal to $85-95 \mathrm{mmHg}$ and CSF pressure $\leq 10 \mathrm{mmHg}$ could be consistently maintained in all patients, allowing clinicians to achieve the target goal of SCP pressure of at least $75-85 \mathrm{mmHg}$. The volume of CSF drainage was consistently less than $50 \mathrm{ml} / \mathrm{h}$ during TEVAR for a maximum volume of $156 \mathrm{ml} /$ day of $\mathrm{CSF}$, as recommended by many $[2,3,12,13,17]$, and the value of MAP was 


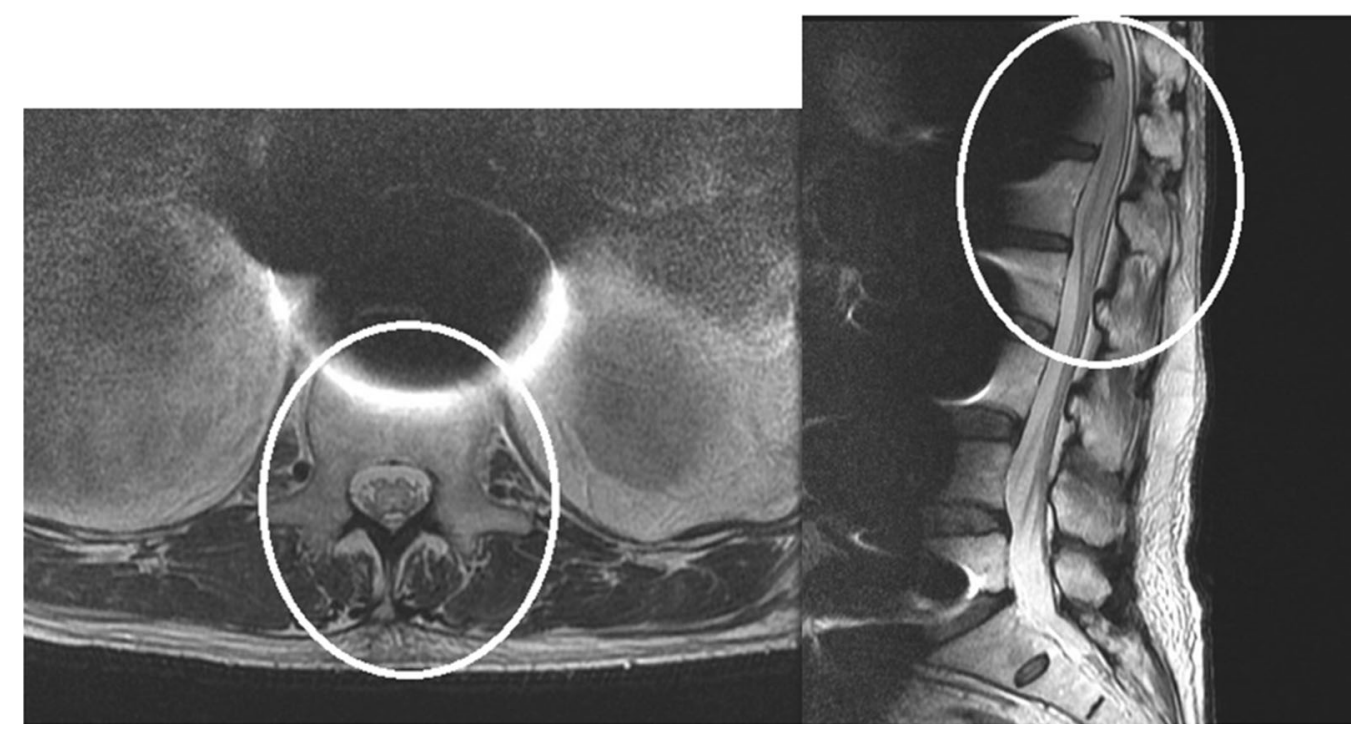

Fig. 3 MRI of the worst case reported. Postprocedure MRI quality was degraded by artifacts from aortic stent (mainly at level of T10 to L1). T2 and FLAIR show central hypo density within lower thoracic spinal cord with some expansion at the level of $\mathrm{T} 10$ to L1, likely representing recent ischemia of spinal cord. The neurological deficits disappeared after $96 \mathrm{~h}$

neurological deficit about 20-24 h after TEVAR and scored in the range of $1-2$. This patient had a total recovery after 5 days. Another patient (Crawford classification type II) had a neurological deficit score between 1 and 2 about $6-8 \mathrm{~h}$ before the TEVAR procedure with deficits in both legs, but more pronounced in the left leg. Five days after TEVAR, this patient had recovered completely. Thus, there were no cases of SCI in this entire population at 5 days postTEVAR.

After the analysis of the results of this protocol, it was decided to compare these data with data obtained from the previous protocol that did not include epidural corticosteroids. While there are statistical and scientific limitations to conclusions drawn from this comparison, it did provide some insight. In fact, when the 47 patients treated with the new protocol were compared to the last 47 consecutive patients treated with the old protocol, taking care to select the same proportion of patients with Crawford's classification types I and II, results show that three patients using the old protocol experienced a neurological deficit (one patient had paraplegia with a score between 1 and 2; the second patient scored between 2 and 3; and classification type II) experienced a delayed 
the third patient had moderate paraparesis, score of 4). This was in contrast with the new protocol using epidurally administered methylprednisolone, where there were no cases of SCI after 5 days.

\section{DISCUSSION}

The ultimate goal of spinal cord protection must be the prevention of paraplegia/paraparesis by using proactive measures. Multimodal protection is essential, especially if long-segment coverage is planned. These strategies should include specific interventions aimed at improving SCI tolerance, reducing the ischemic reperfusion response, and improving oxygen delivery to the spinal cord. This new protocol is based on those key points and allowed our team to be proactive in improving SCI tolerance and reducing ischemia reperfusion response by direct pharmacological treatment with epidurally administered methylprednisolone [28, 32, 46, 50, 51]. Moreover, it optimized oxygen delivery to the spinal cord by implementing a more accurate control and hemodynamic guide and, in that way, improving spinal cord perfusion pressure. Our experience and the results of this new clinical protocol with epidurally administered methylprednisolone have been encouraging. No cases of SCI occurred at 5 days after TEVAR and we have been able to maintain the hemodynamic targets set forth in the protocol. Furthermore, we did not observe any of the complications sometimes associated with epidural steroids [62], spinal drainage [64], and hemodynamic monitoring [52].

The perioperative mortality of three patients with acute complicated TAA cannot be attributed to any iatrogenic causes. The current inhospital mortality rate for acute TAA is high (from $7.7 \%$ to $50 \%$ ) [1, 2, 4, 5, 11, 16, 21, 64], and while the mortality rate for acute TAA in our study is not out of line with current data, it remains suboptimal.

It must be emphasized that our study evaluating this new protocol has limitations, mainly in that it is not a controlled study. The protocol was evaluated in a small population at only two centers. It should be noted there is a paucity of literature on epidural corticosteroids in this setting. The authors found no relevant data regarding the use of epidural corticosteroids in patients undergoing treatment for aortic syndrome (open surgery or endovascular aortic repair). We are not aware of studies comparing patients treated with corticosteroids versus without corticosteroids. Specific data and comparisons on the actual efficacy of systemic corticosteroids are available for trauma $[39,40]$ but not in aortic syndrome.

The effectiveness of any pharmacological treatment for SCI depends in part on the sufficient concentration of drug at the segmental level. From the literature, it is evident that the spinal bioavailability of methylprednisolone after systemic administration is very low [46]. This led to the suggestion of drug administration via intrathecal or epidural routes. Intrathecal drug delivery may not be feasible in aortic repair patients because of their need for lumbar CSF draining. The epidural route appears to be the only reliable option. For that reason, our new protocol evaluated the use of epidurally administered methylprednisolone. There are no data on the use of epidural corticosteroids in the prevention and treatment of SCI and we could find no relevant literature that addressed the intrathecal pharmacokinetics of epidurally administered methylprednisolone in SCI. To the best of our knowledge, epidural steroids are used only in pain management. The majority of studies using epidural steroids to control pain called for doses of epidurally administered methylprednisolone ranging from 20 to $40 \mathrm{mg}$ diluted in a mean volume of $6 \mathrm{ml}$ (3-7 mg of methylprednisolone/ml) for covering three to five spinal segments [61, 62]. The segments involved during TEVAR depend on the type of aneurysm involved and its repair. At the extreme, the segments can span the regions from cervical to lumbar and result in a variety of clinical presentations ranging from vertebrobasilar insufficiency, stroke, arm ischemia, paraparesis, and paraplegia $[1-3,14,22,56]$. On the basis of these data and other studies $[13,50,60-62]$, it was decided that our new protocol would include a high dose of methylprednisolone acetate $(160-200 \mathrm{mg})$ in order to achieve a sufficient local concentration and 
high volume $(25-30 \mathrm{ml})$ to cover all potentially vulnerable segments. The volume and dosage to be injected into the epidural space were, therefore, based on Crawford's classification (vascularization and the medullary segments involved) $[3,61,63,64]$. The volume of diluted drug $(25-30 \mathrm{ml})$, which is the normal volume of anesthetics for epidural anesthesia, injected into the epidural space does not appear to affect the cerebral blood flow (CBF) or spinal cord blood flow (SCBF) in animals with normal intracranial pressure. In pigs with increased intracranial pressure, epidural injection may cause a brief and temporary reduction in CBF and SCBF (with a decrease in cerebral perfusion pressure). Actually, there appears to be a prompt return of $\mathrm{CBF}$ and SCBF to baseline values within about $100-160 \mathrm{~s}$ following epidural injection, likely attributable to autoregulatory mechanisms [65].

This new clinical protocol may serve as a review on how and why clinicians must search for the optimal MAP value ( $\geq 85 \mathrm{mmHg}$ ) and, in so doing, how they may achieve adequate spinal cord perfusion pressure $(\geq 75$ to $80 \mathrm{mmHg}$ ) during TEVAR. In particular, our observations are that using careful, specific hemodynamic monitoring has greatly facilitated an improved approach to achieving the desired MAP values [53-60]. Contrary to open surgery where advanced, complete hemodynamic monitoring is routinely used, precise hemodynamic monitoring is used in only a few protocols for treating TEVAR [13, 60]. In such protocols, $\mathrm{MAP} \geq 80 \mathrm{mmHg}$ was only achieved using basic vital hemodynamic parameters.

In our new clinical protocol with the application of GDT, it is possible to monitor and control hemodynamic parameters (peripheral, vascular, and blood diffusion, i.e., oxygen delivery) which are fundamental to obtaining the right MAP value rather than just achieving a numerical target. For example, in some patients (even normotensives), it might be possible to achieve a MAP $\geq 85 \mathrm{mmHg}$ without full monitoring by working on the hemodynamics, by using a greater quantity of inotropes/vasopressors (particularly noradrenaline) rather than by the correct and precise use of fluids. This means that in some patients, a value of
MAP $\geq 85 \mathrm{mmHg}$ may be noted that may not reflect the right and real physiological value of adequate peripheral tissue perfusion. In other words, this numerical value may be the result of a peripheral shunt and therefore it may be harmful rather than helpful in terms of actual peripheral perfusion (oxygen delivery) of the spinal cord [53-60]. In the event that the patient in this scenario exhibits neurological signs, such a false MAP value might cause the physician to reduce CSF pressure even lower than $10 \mathrm{mmHg}$, greatly enhancing the patient's risk. With the new protocol used in this study, all patients had a CSF pressure maintained consistently at or below $10 \mathrm{mmHg}$.

The results of our initial uncontrolled study of this new protocol have been encouraging, compared with the results present in the literature [66], in particular in that there were no cases of SCI at all. These are also the first data reported on the use of epidural corticosteroids in patients undergoing open or endovascular TAA. It is the hope of the authors that this study and these data may serve as a starting point for continuing future research. Improving TAA outcomes is an urgent and unmet medical need. It may be of interest to study variations on our new protocol such as evaluating the role of epidural corticosteroids administered $12-48 \mathrm{~h}$ prior to a TEVAR procedure.

\section{CONCLUSIONS}

Of the 47 consecutive patients treated with this new protocol at two centers over a 3-year period, none showed SCI 5 days after TEVAR and there were no complications or adverse events. In broad strokes, the protocol calls for CSF drainage, controlled arterial pressure, and the use of epidurally administered methylprednisolone acetate. The protocol requires careful control of hemodynamic parameters, which is not consistently done in endovascular procedures. The use of this new protocol with high dosages of epidurally administered methylprednisolone to help prevent SCI in patients treated with TEVAR has demonstrated clinical feasibility and delivered promising results that warrant further study. 


\section{ACKNOWLEDGEMENTS}

Funding. This paper has been possible thanks to the unconditional support of the Paolo Procacci Foundation, Via Tacito 7, 00193 Roma, Italy. No Rapid Service Fee was received by the journal for the publication of this article.

Medical Writing Assistance. The authors acknowledge medical editing services by Jo Ann LeQuang of LeQ Medical, including Figs. 1 and 2, supported by the Paolo Procacci Foundation funds.

Authorship. All named authors meet the International Committee of Medical Journal Editors (ICMJE) criteria for authorship for this article, take responsibility for the integrity of the work as a whole, and have given their approval for this version to be published.

Author Contributions. Alberto Pasqualucci had the initial idea of the protocol. He, A. AlSibaie, K. Palavilayil Thomas Vaidyan, M. Yousef Nadhari, F. Gori, H. Fawzy Greiss, M. Properzi, O. Sami Maki Al Ani, A. Godwin, A. Hussein Syedkazmi, O. Ahmed Elhanf have studied the cases. A. Paladini and G. Varrassi have analyzed the results. All the authors have contributed to write and ameliorate the quality of the paper. All of them have read and fully accepted the final version of the manuscript.

Disclosures. Giustino Varrassi is a member of the journal's Editorial Board. Antonella Paladini is a member of the journal's Editorial Board. Alberto Pasqualucci, Ayman Al-Sibaie, Kurian Palavilayil Thomas Vaidyan, Mansour Yousef Nadhari, Fabio Gori, Hany Fawzy Greiss, Marina Properzi, Osama Sami Maki Al Ani, Aruna Godwin, Altaf Hussein Syedkazmi and Osama Ahmed Elhanf have nothing to disclose.

Compliance with Ethics Guidelines. The clinical protocol was internally approved by the Dubai Health Authority (see supplementary material). Considering that it was just proposing a different way of administration of wellestablished drugs, approval of the ethics committee was not necessary. All the patients signed an informed consent, specifically indicating that they would have been administered epidural instead of systemic steroids, beside drainage and pressure monitoring of the spinal fluid. They also accepted that their cases could be published, preserving their privacy. The study has been conducted in accordance with the Helsinki Declaration 1964 and its later amendments.

Open Access. This article is distributed under the terms of the Creative Commons Attribution-NonCommercial 4.0 International License (http://creativecommons.org/licenses/ by-nc/4.0/), which permits any noncommercial use, distribution, and reproduction in any medium, provided you give appropriate credit to the original author(s) and the source, provide a link to the Creative Commons license, and indicate if changes were made.

\section{REFERENCES}

1. Erbel R, Aboyans V, Boileau C, et al. 2014 ESC Guidelines on the diagnosis and treatment of aortic diseases: document covering acute and chronic aortic diseases of the thoracic and abdominal aorta of the adult. The Task Force for the Diagnosis and Treatment of Aortic Diseases of the European Society of Cardiology (ESC). Eur Heart J. 2014;35(41):2873-926.

2. Khan SN, Stansby G. Cerebrospinal fluid drainage for thoracic and thoracoabdominal aortic aneurysm surgery. Cochrane Database Syst Rev. 2012;10:CD003635.

3. Awad H, Ramadan ME, El Sayed HF, Tolpin DA, Tili E, Collard CD. Spinal cord injury after thoracic endovascular aortic aneurysm repair. Can J Anaesth. 2017;64(12):1218-35.

4. Badger S, Forster R, Blair PH, Ellis P, Kee F, Harkin DW. Endovascular treatment for ruptured abdominal aortic aneurysm. Cochrane Database Syst Rev. 2017;5:CD005261.

5. Hajibandeh S, Hajibandeh S, Antoniou SA, Torella F, Antoniou GA. Revascularisation of the left subclavian artery for thoracic endovascular aortic repair. Cochrane Database Syst Rev. 2016;4:CD011738. 
6. Svensson LG, Crawford ES, Hess KR, Coselli JS, Safi HJ. Experience with 1509 patients undergoing thoracoabdominal aortic operations. J Vasc Surg. 1993;17(2):357-68 (discussion 368-370).

7. Patel R, Conrad MF, Paruchuri V, Kwolek CJ, Chung TK, Cambria RP. Thoracoabdominal aneurysm repair: hybrid versus open repair. J Vasc Surg. 2009;50(1):15-22.

8. Chiesa R, Tshomba Y, Melissano G, Logaldo D. Is hybrid procedure the best treatment option for thoraco-abdominal aortic aneurysm? Eur J Vasc Endovasc Surg. 2009;38(1):26-34.

9. Melissano G, Bertoglio L, Mascia D, et al. Spinal cord ischemia is multifactorial: what is the best protocol? J Cardiovasc Surg. 2016;57(2):191-201.

10. Gravereaux EC, Faries PL, Burks JA, et al. Risk of spinal cord ischemia after endograft repair of thoracic aortic aneurysms. J Vasc Surg. 2001;34(6):997-1003.

11. Bicknell CD, Riga CV, Wolfe JH. Prevention of paraplegia during thoracoabdominal aortic aneurysm repair. Eur J Vasc Endovasc Surg. 2009;37(6):654-60.

12. Keith CJ Jr, Passman MA, Carignan MJ, et al. Protocol implementation of selective postoperative lumbar spinal drainage after thoracic aortic endograft. J Vasc Surg. 2012;55(1):1-8 (discussion 8).

13. Bobadilla JL, Wynn M, Tefera G, Acher CW. Low incidence of paraplegia after thoracic endovascular aneurysm repair with proactive spinal cord protective protocols. J Vasc Surg. 2013;57(6):1537-42.

14. Winnerkvist A, Anderson RE, Hansson LO, et al. Multilevel somatosensory evoked potentials and cerebrospinal proteins: indicators of spinal cord injury in thoracoabdominal aortic aneurysm surgery. Eur J Cardiothorac Surg. 2007;31(4):637-42.

15. Svensson LG. New and future approaches for spinal cord protection. Semin Thorac Cardiovasc Surg. 1997;9(3):206-21.

16. Safi HJ, Miller CC 3rd, Azizzadeh A, Iliopoulos DC. Observations on delayed neurologic deficit after thoracoabdominal aortic aneurysm repair. J Vasc Surg. 1997;26(4):616-22.

17. Coselli JS, LeMaire SA, Köksoy C, Schmittling ZC, Curling PE. Cerebrospinal fluid drainage reduces paraplegia after thoracoabdominal aortic aneurysm repair: results of a randomized clinical trial. J Vasc Surg. 2002;35(4):631-9.

18. Rathmell JP, Benzon HT, Dreyfuss P, et al. Safeguards to prevent neurologic complications after epidural steroid injections: consensus opinions from a multidisciplinary working group and national organizations. Anesthesiology. 2015;122(5):974-84.

19. Byröd G, Otani K, Brisby H, Rydevik B, Olmarker K. Methylprednisolone reduces the early vascular permeability increase in spinal nerve roots induced by epidural nucleus pulposus application. J Orthop Res. 2000;18(6):983-7.

20. Short DJ, El Masry WS, Jones PW. High dose methylprednisolone in the management of acute spinal cord injury-a systematic review from a clinical perspective. Spinal Cord. 2000;38(5):273-86.

21. Ferrer C, Cao P, De Rango P, Thsomba Y, et al. A propensity-matched comparison for endovascular and open repair of thoracoabdominal aortic aneurysms. J Vasc Surg. 2016;63(5):1201-7.

22. Etz DC, Luehr M, Aspern KV, Misfeld M, et al. Spinal cord ischemia in open and endovascular thoracoabdominal aortic aneurysm repair: new concepts. J Cardiovasc Surg. 2014;55(2 Suppl 1):159-68.

23. Coselli JS, LeMaire SA, Schmittling ZC, Köksoy C. Cerebrospinal fluid drainage in thoracoabdominal aortic surgery. Semin Vasc Surg. 2000;13(4):308-14.

24. Dias-Neto M, Reis PV, Rolim D, Ramos JF, Teixeira JF, Sampaio S. Strategies to prevent TEVAR-related spinal cord ischemia. Vascular. 2017;25(3):307-15.

25. Wong CS, Healy D, Canning C, Coffey JC, Boyle JR, Walsh SR. A systematic review of spinal cord injury and cerebrospinal fluid drainage after thoracic aortic endografting. J Vasc Surg. 2012;56(5):1438-47.

26. Maurel B, Delclaux N, Sobocinski J, et al. The impact of early pelvic and lower limb reperfusion and attentive peri-operative management on the incidence of spinal cord ischemia during thoracoabdominal aortic aneurysm endovascular repair. Eur J Vasc Endovasc Surg. 2015;49(3):248-54.

27. Haulon S, Barillà D, Tyrrell M, Tsilimparis N, Ricotta JJ 2nd. Debate: whether fenestrated endografts should be limited to a small number of specialized centers. J Vasc Surg. 2013;57(3):875-82.

28. Rizvi AZ, Sullivan TM. Incidence, prevention, and management in spinal cord protection during TEVAR. J Vasc Surg. 2010;52(4 Suppl):86s-90s.

29. Crawford ES, Crawford JL, Safi HJ, et al. Thoracoabdominal aortic aneurysms: preoperative and intraoperative factors determining immediate and long-term results of operations in 605 patients. J Vasc Surg. 1986;3(3):389-404. 
30. Oderich G. Current techniques with fenestrated, branched and parallel stent grafts. In: Oderich G, editor. Endovascular aortic repair. Berlin: Springer; 2017.

31. Chou D, Muehle A, Lapchal P, Khoynezhad A. Rabbit spinal cord ischemia model for the development of neuroprotective treatments. In: Zhang J, editor. Neuroprotective therapy for stroke and ischemic disease. Berlin: Springer; 2017. p. 689-700.

32. Wu GJ, Chen WF, Sung CS, et al. Preventive effects of intrathecal methylprednisolone administration on spinal cord ischemia in rats: the role of excitatory amino acid metabolizing systems. Neuroscience. 2007;147(2):294-303.

33. Anderson DK, Means ED, Waters TR, Green ES. Microvascular perfusion and metabolism in injured spinal cord after methylprednisolone treatment. J Neurosurg. 1982;56(1):106-13.

34. Hall ED, Wolf DL, Braughler JM. Effects of a single large dose of methylprednisolone sodium succinate on experimental posttraumatic spinal cord ischemia. Dose-response and time-action analysis. J Neurosurg. 1984;61(1):124-30.

35. Young W, Flamm ES. Effect of high-dose corticosteroid therapy on blood flow, evoked potentials, and extracellular calcium in experimental spinal injury. J Neurosurg. 1982;57(5):667-73.

36. Varrassi G, Marino B, Cosmi E. Influence of methylprednisolone sodium succinate on the microcirculation. Acta Anaesthesiol Ital. 1974;25:159-71.

37. Hall ED. Neuroprotective actions of glucocorticoid and nonglucocorticoid steroids in acute neuronal injury. Cell Mol Neurobiol. 1993;13(4):415-32.

38. Brandolini L, Grannonico M, Bianchini G, Colanardi A, Sebastiani P, Paladini A, Piroli A, Allegretti M. Varrassi G, Di Loreto S. The novel C5aR antagonist DF3016A protects neurons against ischemic neuroinflammatory injury. Neurotox Res. 2019;36:163-74. https://doi.org/10.1007/s12640019-00026-w.

39. Bracken MB, Shepard MJ, Holford TR, et al. Administration of methylprednisolone for 24 or 48 hours or tirilazad mesylate for 48 hours in the treatment of acute spinal cord injury. Results of the third national acute spinal cord injury randomized controlled trial. National Acute Spinal Cord Injury Study. JAMA. 1997;277(20):1597-604.

40. Bracken MB, Shepard MJ, Collins WF, et al. A randomized, controlled trial of methylprednisolone or naloxone in the treatment of acute spinal-cord injury. Results of the second national acute spinal cord injury study. $\mathrm{N}$ Engl J Med. 1990;322(20):1405-11.

41. Evaniew N, Dvorak M. Cochrane in CORR1: steroids for acute spinal cord injury (review). Clin Orthop Relat Res. 2016;474(1):19-24.

42. Miller SM. Methylprednisolone in acute spinal cord injury: a tarnished standard. J Neurosurg Anesthesiol. 2008;20(2):140-2.

43. Qian T, Guo X, Levi AD, Vanni S, Shebert RT, Sipski ML. High-dose methylprednisolone may cause myopathy in acute spinal cord injury patients. Spinal Cord. 2005;43(4):199-203.

44. Matsumoto T, Tamaki T, Kawakami M, Yoshida M, Ando M, Yamada H. Early complications of highdose methylprednisolone sodium succinate treatment in the follow-up of acute cervical spinal cord injury. Spine. 2001;26(4):426-30.

45. Pointillart V, Petitjean ME, Wiart L, et al. Pharmacological therapy of spinal cord injury during the acute phase. Spinal Cord. 2000;38(2):71-6.

46. Koszdin KL, Shen DD, Bernards CM. Spinal cord bioavailability of methylprednisolone after intravenous and intrathecal administration: the role of P-glycoprotein. Anesthesiology. 2000;92(1):156-63.

47. Sekhon LH, Fehlings MG. Epidemiology, demographics, and pathophysiology of acute spinal cord injury. Spine. 2001;26(24 Suppl):S2-12.

48. Reece TB, Kern JA, Tribble CG, Cassada DC. The role of pharmacology in spinal cord protection during thoracic aortic reconstruction. Semin Thorac Cardiovasc Surg. 2003;15(4):365-77.

49. Braughler JM. Lipid peroxidation-induced inhibition of gamma-aminobutyric acid uptake in rat brain synaptosomes: protection by glucocorticoids. J Neurochem. 1985;44(4):1282-8.

50. Quirno M, Campbell K, Yoo A, et al. The effect of prophylactic local steroid delivery in a spinal cord injury model. Spine J. 2012;12:88S.

51. Rose FX, Estebe JP, Ratajczak M, et al. Epidural, intrathecal pharmacokinetics, and intrathecal bioavailability of ropivacaine. Anesth Analg. 2007;105(3):859-67.

52. Varrassi G, Guadagni S, Ciccozzi A, et al. Hemodynamic variations during thoracic and abdominal stop-flow regional chemotherapy. Eur J Surg Oncol. 2004;30(4):377-83.

53. Giglio M, Manca F, Dalfino L, Brienza N. Perioperative hemodynamic goal-directed therapy and 
mortality: a systematic review and meta-analysis with meta-regression. Minerva Anestesiol. 2016;82(11):1199-213.

54. Cecconi M, Corredor C, Arulkumaran N, et al. Clinical review: goal-directed therapy-what is the evidence in surgical patients? The effect on different risk groups. Crit Care. 2013;17(2):209.

55. Benes J, Pradl R, Chytra I. Perioperative hemodynamic optimization: a way to individual goals. In: Vincent J-L, editor. Annual update in intensive care and emergency medicine. Berlin: Springer; 2012.

56. Cecconi M, Correcdor C, Rhodes A. Perioperative goal-directed therapy: monitoring, protocolized care, and timing. In: Vincent J-L, editor. Annual update in intensive care and emergency medicine. Berlin: Springer; 2012. p. 368-82.

57. Lobo SM, de Oliveira NE. Clinical review: what are the best hemodynamic targets for noncardiac surgical patients? Crit Care. 2013;17(2):210.

58. Benes J, Giglio M, Brienza N, Michard F. The effects of goal-directed fluid therapy based on dynamic parameters on post-surgical outcome: a meta-analysis of randomized controlled trials. Crit Care. 2014;18(5):584.

59. Vincent JL, Rhodes A, Perel A, et al. Clinical review: update on hemodynamic monitoring-a consensus of 16. Crit Care. 2011;15(4):229.
60. Acher C, Acher CW, Marks E, Wynn M. Intraoperative neuroprotective interventions prevent spinal cord ischemia and injury in thoracic endovascular aortic repair. J Vasc Surg. 2016;63(6):1458-65.

61. Bromage PR. Spread of analgesic solutions in the epidural space and their site of action: a statistical study. Br J Anaesth. 1962;34:161-78.

62. Pasqualucci A, Varrassi G, Braschi A, et al. Epidural local anesthetic plus corticosteroid for the treatment of cervical brachial radicular pain: single injection versus continuous infusion. Clin J Pain. 2007;23(7):551-7.

63. Frederick JR, Woo YJ. Thoracoabdominal aortic aneurysm. Ann Cardiothorac Surg. 2012;1(3):277-85.

64. Wynn MM, Mell MW, Tefera G, Hoch JR, Acher CW. Complications of spinal fluid drainage in thoracoabdominal aortic aneurysm repair: a report of 486 patients treated from 1987 to 2008. J Vasc Surg. 2009;49(1):29-34 (discussion 34-35).

65. Grocott HP, Mutch WA. Epidural anesthesia and acutely increased intracranial pressure. Lumbar epidural space hydrodynamics in a porcine model. Anesthesiology. 1996;85(5):1086-91.

66. Heldner MR, Arnold M, Nedeltchev K, Gralla J, Beck J, Fisher U. Vascular diseases of the spinal cord: a review. Curr Treat Options Neurol. 2012;14(6):509-20. https://doi.org/10.1007/s11940-012-0190-9. 